\title{
Transjugular Intrahepatic Portosystemic Shunt Reduction for Medically Refractory Hepatic Encephalopathy \\ Brandon Toliver ${ }^{1}$, Adam Schmitz, ${ }^{2 ; 3}$ Paul Haste $^{2}$ \\ ${ }^{1}$ Indiana University School of Medicine \\ ${ }^{2}$ Indiana University School of Medicine, Department of Radiology \\ ${ }^{3}$ Ascension St. Vincent Hospital, Department of Internal Medicine
}

Background: Transjugular intrahepatic portosystemic shunt (TIPS) is an established intervention for symptomatic portal hypertension. Following TIPS creation, $22-50 \%$ of patients experience hepatic encephalopathy ( $\mathrm{HE}$ ), with symptoms ranging from mild confusion to coma. While HE can be medically managed, refractory cases may require downsizing of the TIPS which can be accomplished by deploying a smaller caliber stent within the original shunt. Decreasing shunt diameter redirects blood flow back through liver parenchyma. The purpose of this study was to evaluate the efficacy of TIPS downsizing for the treatment of medically refractory post-TIPS HE.

Methods: An IRB-approved, HIPAA compliant retrospective review was performed. A search of an institutional radiology database yielded 45 patients who underwent TIPS downsizing between 2011-2021. Four patients were excluded due to lack of post-TIPS HE, and a total 41 patients were included in the study. Clinical and serologic data were obtained for all 41 patients. The primary objective was to determine the efficacy of TIPS downsizing for reduction of HE as measured by the West Haven criteria. Secondary endpoints included post-downsize recurrence of ascites or gastrointestinal bleeding, procedural complications, and thirty-day mortality.

Results: TIPS downsizing was performed in all 41 patients with a $9.8 \%$ thirty-day mortality rate. No deaths were attributable to the procedure itself. Of the remaining patients, twenty-seven patients $(65.9 \%)$ had improvement in HE and 10 patients $(24.4 \%)$ proceeded to TIPS occlusion due to refractory HE. The average pre-downsize and post-downsize West Haven grades were $2.9 \pm 0.5$ and $1.9 \pm 1.2$, respectively. One patient $(2.4 \%)$ had spontaneous TIPS thrombosis after downsizing and developed gastrointestinal bleeding requiring embolization; 15 patients (36.6\%) experienced recurrent ascites.

Conclusion: In this population, TIPS downsizing improved medically refractory HE in $65.9 \%$ of patients with a $2.4 \%$ risk of recurrent gastrointestinal bleeding, $36.6 \%$ risk of recurrent ascites, and $9.8 \%$ thirty-day mortality. 